

\title{
System-Oriented Reliability-Based Methodology for Optimal Joint Maintenance and Production Planning
}

I. Roda, M. Macchi, C. Parmigiani, A. A. Arata

\section{To cite this version:}

I. Roda, M. Macchi, C. Parmigiani, A. A. Arata. System-Oriented Reliability-Based Methodology for Optimal Joint Maintenance and Production Planning. IFIP International Conference on Advances in Production Management Systems (APMS), Sep 2017, Hamburg, Germany. pp.92-100, 10.1007/9783-319-66923-6_11 . hal-01666159

\section{HAL Id: hal-01666159 \\ https://hal.inria.fr/hal-01666159}

Submitted on 18 Dec 2017

HAL is a multi-disciplinary open access archive for the deposit and dissemination of scientific research documents, whether they are published or not. The documents may come from teaching and research institutions in France or abroad, or from public or private research centers.
L'archive ouverte pluridisciplinaire HAL, est destinée au dépôt et à la diffusion de documents scientifiques de niveau recherche, publiés ou non, émanant des établissements d'enseignement et de recherche français ou étrangers, des laboratoires publics ou privés. 


\title{
System-oriented reliability-based methodology for optimal joint maintenance and production planning
}

\author{
Roda I. ${ }^{1}$, Macchi M. ${ }^{1}$, Parmigiani C. ${ }^{1}$, Arata A.A. ${ }^{2}$ \\ ${ }^{1}$ Politecnico di Milano, Piazza Leonardo da Vinci, Milano, Italy \\ ${ }^{2}$ Pontificia Universidad Católica de Valparaíso, Av. Brasil 2950, Valparaíso, Chile \\ \{irene.roda, marco.macchi, carlo.parmigiani\}@polimi.it \\ adolfo.arata@ucv.cl
}

\begin{abstract}
The integration among the organizational functions managing assets along its lifecycle is a crucial aspect for production companies to implement Asset Management. In this paper, an iterative four-step methodology is presented to support the joint maintenance and production planning considering the system configuration optimization as well. The objective is to overcome the limitations of most of the approaches that can be found in the literature regarding joint optimization models, by integrating it with a system-oriented and reliabilitybased approach. Reliability, Maintainability and Availability analysis at system level is used to support the traditional joint optimization models. The methodology is applied in an industrial case in the mining sector.
\end{abstract}

Keywords: Joint maintenance and production planning, asset management, lifecycle, production system, reliability.

\section{Introduction}

One of the core concepts within the Asset Management discipline is the multidisciplinary integration among functions in an organization to manage assets along its lifecycle [1], [2]. Within this perspective, the typical 'silo' behaviour which keeps maintenance and other life cycle processes separated in an organization is no longer sustainable [1], [3], [4]. In particular, when referring to production contexts, maintenance and operations are the two functions dealing directly with the assets to make it operate and that need to work in an integrated way. Nevertheless, the integration between these two functions is still a challenging issue for companies. In fact, they are related through a strict but apparently contrasting interconnection, dealing with the same resources (production assets) with different goals [5]. On one side, the Availability of a plant is connected to the effectiveness of maintenance activities that should be capable to minimize the amount of time in which the plant is in a fault state. On the other hand, the Utilization is related to the production activity: the plant is utilized to reach commercial objectives considering the resource constrains. The contrast between maintenance and production functions is related to the unreliable nature of the machines that compose a system. When asset performances diverge from 
its desired target value, the interest of maintenance is to stop the asset, in order to intervene to extend its life cycle availability, while production needs to utilize it to maximize its efficiency. For this reason, there is the need of finding proper models that enable a joint planning of maintenance and production, which are based on a reliability approach [6]. Moreover, our hypothesis is that such models should address asset systems in their entirety, taking into account that industrial assets are complex systems composed by different components interrelating among themselves. Such interactions, together with the state of each component, affect the state and performance of the system itself. Hence, the effect of any local decision has to be considered in maintenance and production joint planning [7]. "Effective life cycle management is one of the key responsibilities of production and maintenance management at the asset system level" [3]. Successfully integrating the planning of production and maintenance activities can bring several benefits in terms of efficiency of the production system. The idea is to determine a global optimum avoiding to reach a local optimum that maximize the performance of a single function affecting negatively the performance of the other.

The production system design is a critical aspect to be considered as well, since the design decisions may have a decoupling effect between maintenance and production. In fact, optimal design is capable to provide a spare capacity that can reduce the impact of the maintenance intervention over the system production and can reduce the demand for maintenance, increasing the reliability of the system with redundant resources.

The aim of this paper is to present a methodology that support reliability-based system-oriented joint optimization of maintenance and production planning, keeping into account alternative configuration scenarios as well. Section 2 is focused on the State of art on the addressed topic. The developed methodology is presented in Section 3. In Section 4 the application of the methodology in a real industrial context in the mining sector is presented and discussed. Finally, Section 5 concludes the paper.

\section{State of art and problem statement}

In the scientific literature, it is possible to find several reviews about joint optimization models such as [8], [9]. Overall, by investigating the models that have been proposed so far, several existing limitations can be identified, and the main ones are the following. Firstly, many of them do not truly implement real integration among maintenance and production planning but they use sequential or hierarchical approach [10]. Secondly, few models take into account the systemic perspective and the relevance of the design configuration in the optimization process. In fact, a very common approach is the so called black box modelling, where it is assumed that the performance of single machines perfectly reflects the systemic one. In the literature, some models propose alternative solutions taking into account this aspect [11], [12], [13]. Even if those models take into account the necessity of considering the occurring interrelationship between system components, the complete systemic vision is still missing. Finally, most models are characterized by low degree of applicability, being mathematical models with high computational complexity; losing its usefulness in industrial implementation [8]. 
Overall, the gap in the literature targeted in this paper is the absence of a methodology that, without increasing the computational effort of the models, integrates the planning of maintenance and production, and the optimal design configuration of a system. To reach this objective, the methodology must keep: i) systemic perspective, considering the impact of all the elements in the system; ii) reliability-based approach, considering the uncertainty of machines behaviour; iii) life cycle orientation, supporting decisions along the asset life cycle [14].

\section{System-oriented reliability-based methodology for joint maintenance and production planning}

The methodology proposed in this paper aims to integrate the joint optimization models that can be found in the literature, with a systemic perspective evaluating the optimization process at system level based on a reliability-oriented approach. The methodology is based on the premise that, since it is fundamental to keep a systemic perspective, the relationship between the design of the system and maintenance and production planning has to be considered as well.

An iterative four-step methodology is proposed that integrates a joint optimization model with Reliability and Maintenance engineering analysis at system level. It is based on the modelling of the system under analysis through the Reliability Block Diagram (RBD) technique offering an integrated and reliability-oriented view of the system with a bottom-up perspective while keeping an easy implementation approach [15]. Stochastic simulation is then used for describing the random nature of reliability-related phenomena [16]. This approach has been exploited in the defined methodology in order to effectively identify the criticalities in the system, and therefore the improvement opportunities. The Joint optimization model is integrated with it and is applied at unitary process level (defined as the group of system components used for a macrophase of the production process) (Figure 1).



Fig. 1. System hierarchy representation

The output of the methodology consists of a joint production and maintenance planning and of the indication of the optimal design solution at system level. In particular, the methodology is composed of four steps and they are: i) system criticality analysis, ii) configuration alternatives definition, iii) joint optimization model resolution, iv) reliability-based system-level performance analysis. Each step is detailed hereafter. 
STEP 1: System criticality analysis. The first step aims at implementing performance analysis at system level for detecting criticalities. The main objective is to calculate the incidence of each system component on the overall system availability, taking into account its performance and its positioning within the RBD model of the system. Monte Carlo simulation is used to run the performance analysis and the most critical unitary processes and components are identified. This step enables focusing on the criticalities.

STEP 2: Configuration alternatives definition. In the second step, a discrete set of configuration alternatives is defined. In practice, the installation of redundancies (total redundancy, stand-by etc.) to the most critical components may be considered. In doing so, both the reliability perspective and the technical feasibility must be considered. Once the set of alternatives is defined, the expected performances for each of it are evaluated by modifying the RBD model accordingly and running the simulation. In this way, the input to the next step is given by a set of configuration alternatives for each critical unitary process with the related expected performance and total cost of ownership (related CAPEX and expected OPEX). The configuration alternatives together with the as-is scenario are the input for the next optimization step.

STEP 3: Joint optimization model resolution. The third step consists in running a joint optimization model to determine the decision variables optimal values. The following outputs are generated given a reference time horizon: production planning in terms of number of batches to be produced in each time interval, maintenance planning in terms of frequency of preventive maintenance interventions and selection of optimal configuration alternative among the ones identified in step 2, for each critical unitary process under analysis.

STEP 4: Reliability-based system-level performance analysis. The last step allows going back from the unitary process level to the system level. The aim of this step is to evaluate if the optimization outputs in terms of configuration alternatives effectively improve the overall performance of the system. In order to do so, the reconfiguration options selected by the joint optimization model for the critical unitary processes are integrated in the system RBD model and the overall system performances are evaluated through simulation and can be compared with the 'as is' scenario's performances.

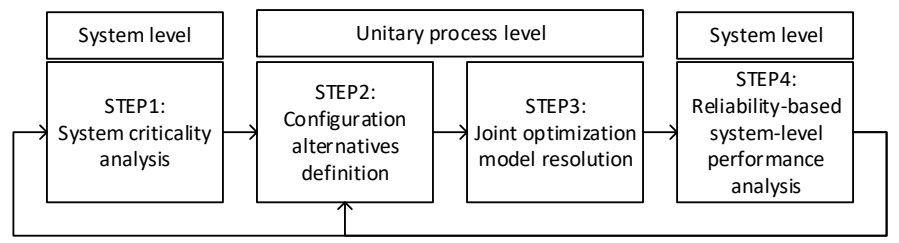

Fig. 2. The proposed methodology

A recursive approach is proposed. In fact, the reconfiguration options from the optimization model at unitary process level may not affect the system performance. In this case, it is possible to go back to step 2. Moreover, in case that a reconfiguration option is identified, new improvement opportunities can be found by running again the methodology from step 1 selecting criticalities in the new system configuration. 


\section{Application Case}

The proposed methodology was tested in an application case in the mining industry. The analyzed plant is a top-class copper concentrate production plant located in Chile. A model capable to deal with the structure of a batch process system, characterized by the presence of various inter-operational buffers, was selected from the literature [12]. Fig. 3 shows the production process under analysis, showing the unitary processes composing it. The asset under analysis is at its Middle of Life stage.

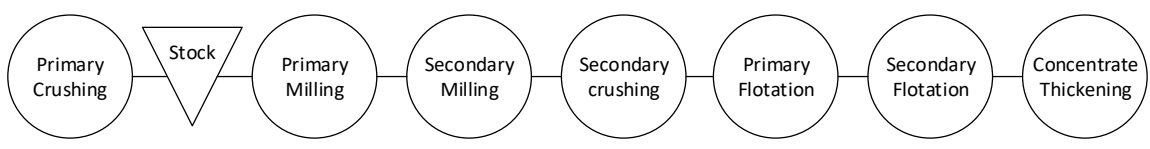

Fig. 3. Production process scheme at Unitary Process level

As supporting tools, the Reliability and Maintenance Engineering Software, R-MES Project $\bigodot$ (developed by the CGS company http://www.cgssa.com/en/) is used in order to implement the performance analysis at steps 1,2 and 4, supporting RBD modelling and Monte Carlo simulation. An optimization solver for determining the solutions of the joint optimization model for maintenance and production planning used at step 3 .

STEP 1: System criticality analysis. By building the RBD model of the plant and running Monte Carlo simulation, it is possible to identify which of the unitary processes impact more on the system performance (availability). It resulted that primary crushing, primary milling, secondary milling and secondary crushing account for $80 \%$ of the system unavailability. The following steps are applied to these four unitary processes. Moreover, breaking down the performance analysis within each critical unitary process it was possible to identify the criticalities at component level (critical equipment).

STEP 2: Configuration alternatives definition. Considering that the mining context does not present strong constrains in terms of financial and space availability, reconfiguration alternatives were defined for each critical unitary process identified at step 1. Table 1 shows the set of reconfiguration alternatives for the primary crushing. Performance analysis was run through simulation, based on the modified RBD model of the system integrating the modification required by each alternative. The output is the expected failure rate and related CAPEX for each configuration alternative.

Table 1. Reconfiguration alternatives for the primary crushing unitary process

\begin{tabular}{lccl}
\hline $\begin{array}{c}\text { Reconfiguration } \\
\text { alternatives }\end{array}$ & $\begin{array}{c}\text { Expected } \\
\text { failure rate }\end{array}$ & $\begin{array}{c}\text { CAPEX } \\
{[\mathbf{k} \mathbf{\$}]}\end{array}$ & \multicolumn{1}{c}{ Proposed reconfiguration } \\
\hline 'As-Is' & 0,0026 & 0 & No change in the system \\
'To-be 1' & 0,0015 & 6,25 & Add a redundancy over the Crusher \\
'To-be 2' & 0,0014 & 7,1 & Add a redundancy on Overload-Feeder line \\
\hline
\end{tabular}


STEP 3: Joint optimization model resolution. In this step, the optimization model [12] was applied for each unitary process considering the different configuration alternatives. The joint model's objective function is the maximization of the system effectiveness, that is given by the difference between the system expected revenues and the required investments cost and the production and maintenance costs (both hidden and tangible) in the planning horizon. The output is the optimal joint planning of maintenance and production, the expected utilization and availability (Fig. 4), the number of batches to be produced in each period and the batch size. Moreover, the reconfiguration alternatives that maximize the objective function are selected for each unitary process under analysis (by mean of a Boolean variable) (Table 2).

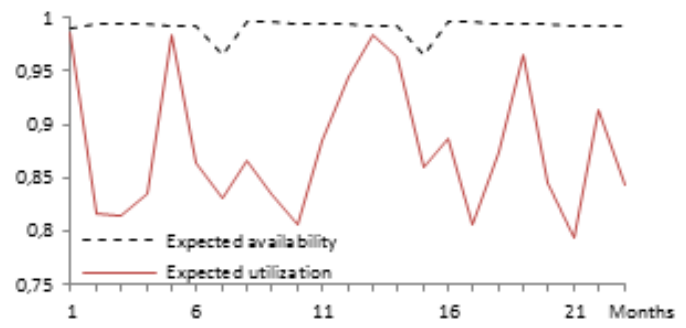

Fig. 4. Joint optimization output: expected utilization and availability of the plant

Table 2. Joint optimization output: optimal configuration alternative for each unitary process

\begin{tabular}{c|ccc}
\cline { 2 - 4 } & \multicolumn{3}{c}{ Configuration Alternatives } \\
\hline Critical unitary processes & 'As-is' & 'To-be 1' & 'To-be 2' \\
\hline Primary Crushing & 0 & $\mathbf{1}$ & 0 \\
Primary Milling & 0 & 0 & $\mathbf{1}$ \\
Secondary Milling & $\mathbf{1}$ & 0 & 0 \\
Secondary Crushing & 0 & $\mathbf{1}$ & 0 \\
\hline
\end{tabular}

STEP 4: Reliability-based system-level performance analysis. The selected reconfiguration alternatives come from the optimization done at unitary process level in the previous step. In order to evaluate if the system performance effectively improves by introducing them, system performance analysis is run again through simulation and adjusting the RBD model of the plant. In this case, the expected system unavailability is reduced from $18 \%$ to $10 \%$ justifying a reconfiguration investment's profitability.

\subsection{Discussion}

The joint framework application enlightens the potentiality of the integration between system design and maintenance and production planning. Scenario-based sensitivity analysis is useful to discuss the underlying dynamics that correlate maintenance and production planning with system design. Table 3 shows how the design reconfiguration selected through the joint optimization model changes when MTTR (Mean Time to Repair) variates. Reducing the MTTR, the sub-systems criticality decreases, due to lower expected hidden costs and therefore investments in design decrease as well. 
Table 3. Optimal configuration alternatives



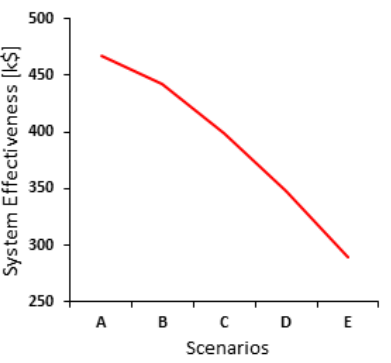

Fig. 5. Sensitivity analysis: System effectiveness vs MTTR reduction

Another scenario-based sensitivity analysis was conducted considering the system production capacity (Table 4 ).

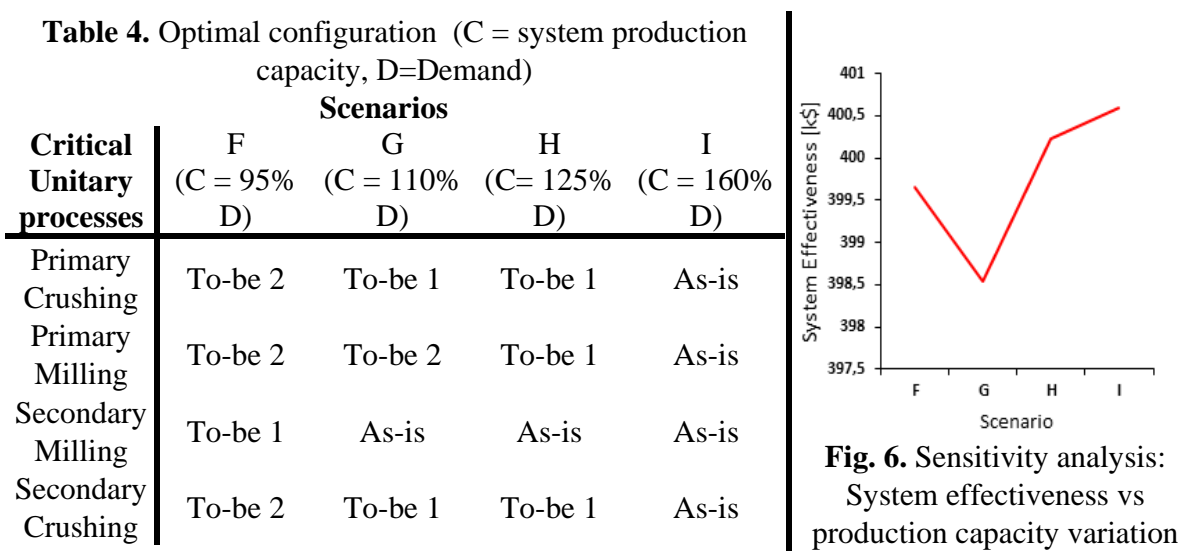

The design configurations selected through the joint optimization model in the scenarios enlighten how required investments in new equipment are lower when the spare capacity is higher than the demand (in the extreme scenario (I) the investments result null). Moreover, in that case, the model indicates higher maintenance frequency.

\section{Conclusions}

The integration between maintenance and production planning and design reconfiguration is a challenging aspect for asset management. The methodology proposed in this paper introduces the possibility to integrate existing joint optimization models in the literature with a system-oriented reliability-based approach. The methodology consists in a four-step iterative process, which, through a reliabilityoriented approach, enables evaluating a system's criticalities, identifying the best design reconfiguration alternative and determining the optimal maintenance and production planning. The methodology is applied in the mining industry. 


\section{Acknowledgments}

The research work was performed within the context of SustainOwner ("Sustainable Design and Management of Industrial Assets through Total Value and Cost of Ownership"), a project sponsored by the EU Framework Programme Horizon 2020, MSCA-RISE-2014: Marie Skłodowska-Curie Research and Innovation Staff Exchange (RISE) (grant agreement number 645733-Sustain-Owner- H2020-MSCA-RISE-2014).

\section{References}

[1] J. E. Amadi-Echendu, R. Willett, K. Brown, "What is engineering asset management?" Definitions, Concepts and Scope of Engineering Asset Management, Springer London, 2010, pp. 3-16.

[2] J.S.Mitchell, Physical Asset Management Handbook. Clarion Tech. Publishers, 2002.

[3] CEN/TC 319/WG 10, "Maintenance within physical asset management," 2014.

[4] M. Gram and S. Werner, "Evaluating the life cycle costs of plant assets: A multidimensional view," Serbian J. Manag., vol. 7, no. 2, pp. 287-298, 2012.

[5] U. M. Al-Turki, B. S. Yilbas, and A. Z. Sahin, "Maintenance in Manufacturing Environment: An Overview," Integrated Maint. Planning in Manuf. Systems. 2014.

[6] U. M. Al-Turki, T. Ayar, B. S. Yilbas, and A. Z. Sahin, "Integrated Maintenance Planning,” Integr. Maint. Plan. Manuf. Syst., pp. 25-57, 2014.

[7] I. Roda, M. Macchi, "Studying the funding principles for integrating Asset Management in Operations: an empirical research in production companies," IFACPapersOnLine, vol. 49, no. 28, pp. 1-6, 2016.

[8] G. Budai, R. Dekker, R. P. Nicolai, "Maintenance and Production: A Review of Planning Models," Complex System Maintenance Handbook, Springer London, 2008

[9] D. Pandey, M. S. Kulkarni, P. Vrat, "Joint consideration of production scheduling, maintenance and quality policies: a review and conceptual framework," Int. J. Adv Oper. Manag., vol. 2, p. 1, 2010.

[10] F. Benbouzid, C. Varnier, and N. Zerhouni, "Resolution of joint maintenance/ production scheduling by sequential and integrated strategies," Artif. Neural Nets Probl. Solving Methods, vol. 2687/2003, pp. 782-789, 2003.

[11] E. N. Pistikopoulos, C. G. Vassiliadis, J. Arvela, and L. G. Papageorgiou, "Interactions of maintenance and production planning for multipurpose process plants - a system effectiveness approach," Ind. Eng. Chem. Res., vol. 40, pp. 3195-3207, 2001.

[12] H. D. Goel, J. Grievink, and M. P. C. Weijnen, "Integrated optimal reliable design, production, and maintenance planning for multipurpose process plants," Comput. Chem. Eng., vol. 27, no. 11, pp. 1543-1555, 2003.

[13] N. Nahas and M. Nourelfath, "Buffer allocation, machine selection and preventive maintenance optimization in unreliable production lines," Proc. 2015 Int. Conf. Ind. Eng. Syst. Manag. IEEE IESM 2015, no. October, pp. 1028-1033, 2016.

[14] K. Shahata and T. Zayed, "Simulation as a tool for life cycle cost analysis," in Proceedings of the 40th Conference on Winter Simulation, 2008, pp. 2497-2503.

[15] I. Roda and M. Garetti, "Application of a performance - driven TCO evaluation model for physical asset management," in 9th WCEAM Research Papers, 2015, pp. 11-23.

[16] M. Macchi, F. Kristjanpoller, M. Garetti, A. Arata, and L. Fumagalli, "Introducing buffer inventories in the RBD analysis of process production systems," Reliab. Eng. Syst. Saf., vol. 104, pp. 84-95, Aug. 2012. 